\title{
Experimental evaluation on surface plasmon resonance sensor performance based on sensitive hyperbranched polymer nanocomposite thin films
}

\begin{abstract}
The plethora of optical techniques have been developed to detect the dengue virus, regrettably, the existing techniques are cumbersome and have limitations to provide early identification of the lowlevel of dengue virus. For this purpose, surface plasmon resonance (SPR) optical sensor was designed with a highly sensitive thin film based on hyperbranched polymer nanocomposites for conjugation of antibodies that specifically detect the dengue virus type II (DENV). In particular, we investigate the SPR sensing performance for gold/cadmium sulfide quantum dotspolyamidoamine/antibody (Au/CdS QDs-PAMAM/IgM) sensor film in terms of linear sensitivity, binding affinity, detection accuracy, full-width-half-maximum, selectivity, and spike and recovery test. The results show a strong affinity value, good sensitivity and selectivity, high accuracy, and excellent recovery value, obeying the ideal value based on conventional assay analysis. Additionally, this Au/CdS QDs-PAMAM/IgM based SPR sensor was successfully applied for the lowest detection of $1 \mathrm{pM}$ of DENV E-proteins. To this end, a highly sensitive Au/CdS QDsPAMAM/IgM sensor film was characterized using Raman spectroscopy, ultraviolet-visible (UVVis-NIR) spectroscopy, and atomic force microscopy (AFM) to confirm the binding of DENV.
\end{abstract}

Keyword: Surface plasmon resonance; Optical sensor chip; Dengue virus; Polyamidoamine dendrimer; Thin film 